\title{
A Novel Cyclosporin A Aqueous Formulation for Dry Eye Treatment: In Vitro and In Vivo Evaluation
}

\author{
Claudia Di Tommaso, ${ }^{1}$ Fatemeb Valamanesh ${ }^{2,3}$ Florence Miller, ${ }^{1}$ Pascal Furrer, ${ }^{1}$ Marta \\ Rodriguez-Aller, ${ }^{1}$ Francine Behar-Cohen, ${ }^{2,4}$ Robert Gurny, ${ }^{1}$ and Michael Möller ${ }^{1}$
}

Purpose. The aim of the present study was the in vitro and in vivo evaluation of a novel aqueous formulation based on polymeric micelles for the topical delivery of cyclosporine A for dry eye treatment.

Methods. In vitro experiments were carried out on primary rabbit corneal cells, which were characterized by immunocytochemistry using fluorescein-labeled lectin I/isolectin B4 for the endothelial cells and mouse monoclonal antibody to cytokeratin $3+12$ for the epithelial ones. Living cells were incubated for 1 hour or 24 hours with a fluorescently labeled micelle formulation and analyzed by fluorescence microscopy. In vivo evaluations were done by Schirmer test, osmolarity measurement, CyA kinetics in tears, and CyA ocular distribution after topical instillation. A $0.05 \%$ CyA micelle formulation was compared to a marketed emulsion (Restasis).

Results. The in vitro experiments showed the internalization of micelles in the living cells. The Schirmer test and osmolarity measurements demonstrated that micelles did not alter the ocular surface properties. The evaluation of the tear fluid gave similar CyA kinetics values: AUC $=2339 \pm 1032 \mathrm{~min}^{*} \mu \mathrm{g} / \mathrm{mL}$ and $2321 \pm 881.63 ; \mathrm{Cmax}=478 \pm 111 \mu \mathrm{g} / \mathrm{mL}$ and $451 \pm 74$; half-life $=36 \pm 9 \mathrm{~min}$ and $28 \pm 9$ for the micelle formulation and Restasis, respectively. The ocular distribution investigation revealed that the novel formulation delivered $1540 \pm 400 \mathrm{ng}$ CyA/g tissue to the cornea.

Concuusions. The micelle formulation delivered active CyA into the cornea without evident negative influence on the ocular surface properties. This formulation could be applied for immune-related ocular surface diseases. (Invest Ophthalmol Vis Sci. 2012;53:2292-2299) DOI:10.1167/iovs.11-8829

D ry eye is a multifactorial disease, with two major recognized forms: one is characterized by a reduction of tear production, and the other is characterized by tear hyperosmolarity, mainly due to excessive evaporative water loss. ${ }^{1,2}$ These two components of dry eye engender inflammation and ocular surface irritation. Thus, the goals for the

${ }^{1}$ School of Pharmaceutical Sciences, University of Geneva, University of Lausanne, 30 Quai Ernest Ansermet, CH-1211 Geneva 4, Switzerland. 'INSERM, UMRS 872 Team 17, Centre de Recherches des Cordeliers, 15 rue de l'école de médecine, 75006 Paris, France. ${ }^{3}$ Fondation A. De Rothschild, 25 rue Manin, 75019 Paris, France. ${ }^{4}$ Université Paris Descartes, Faculté de médecine, Département d'ophtalmologie, Hôpital Hôtel-Dieu, APHP, Paris, France.

Submitted for publication October 20, 2011; revised January 23, 2012; accepted February 26, 2012.

Disclosure: C. Di Tommaso, None; F. Valamanesh, None; F. Miller, None; P. Furrer, None; M. Rodriguez-Aller, None; F. Behar-Cohen, None; R. Gurny, None; M. Möller, None

Corresponding author: Michael Möller, Tel: +41 22379 3132, Fax: +41223796567, Michael.Moeller@unige.ch. treatment of this disease are to improve the patient's ocular comfort and to return the ocular surface and tear composition to their basal and healthy states. Two main therapeutic approaches are used in the clinic: instillation of artificial tears for tear supplementation and stimulation and instillation of anti-inflammatory drugs to reduce ocular surface inflammation. ${ }^{3}$ Inflammation can be reduced by the use of corticosteroids, tetracyclines or cyclosporin A. For CyA, the mechanism of action of how tear production is increased is not totally clear, but it seems to be related to its immuno-modulatory activity, which decreases the local inflammation. ${ }^{3}$ CyA is a neutral, cyclic undecapeptide with many pharmacological activities: suppression of T-cell-mediated responses, inhibition of chronic inflammatory reactions, fungicidal activity, and antihepatitis $\mathrm{C}$ virus activity. ${ }^{4}$ This drug has been extensively used to suppress the alloimmune response after solid organ transplantation since the 1970s. Other applications of CyA are the treatment of ophthalmic diseases such as dry eye syndrome and autoimmune uveitis, and the prevention of corneal graft rejection. ${ }^{5}$ CyA has also been investigated for treating several eye infections, such as posterior blepharitis, ${ }^{6}$ atopic keratoconjunctivitis, ${ }^{7}$ and herpetic stromal keratitis. ${ }^{8}$ For many of these diseases, high systemic concentrations of CyA have to be administered to reach therapeutic ocular drug levels (50-300 $\left.\mathrm{ng} \mathrm{CyA}_{\mathrm{A}} / \mathrm{g}_{\text {tissue }}\right){ }^{9}$ resulting in serious side effects, such as nephrotoxicity and hypertension. ${ }^{4}$ Hence, a topical and local CyA administration would be favorable to directly target the disease site, especially for the ocular surface treatment in dry eye. However, due to its high hydrophobicity $(\log \mathrm{P}$ octanol/water $=8.2)$ and its low water solubility at $25^{\circ} \mathrm{C}$ $(0.012 \mathrm{mg} / \mathrm{mL}),{ }^{10} \mathrm{CyA}$ is difficult to formulate into a topical aqueous solution at an effective concentration. Despite many research efforts, only one emulsion for dry eye treatment has reached the US market: Restasis (Allergan, Irvine, CA). This formulation reduces the disease symptoms by decreasing activated lymphocytes. ${ }^{11}$ However, it has drawbacks, such as burning and stinging sensations. ${ }^{12}$ To decrease local side effects and to enhance the patient's comfort, CyA colloidal drug delivery systems are an interesting option for formulations to enable them to overcome ocular barriers. ${ }^{13-16}$

The present work is focused on a novel topical micelle formulation based on methoxy poly(ethylene) glycol (MPEG)hexyl-substituted poly(lactides) (hexPLA) to treat immunerelated ocular diseases and, in particular, dry eye syndrome. The formulation presented here has already shown excellent in vitro and in vivo ocular biocompatibility and efficiency in CyA solubilization. ${ }^{10,16}$ Here, in vitro and in vivo tests were performed to evaluate the suitability of this micelle formulation for dry eye treatment. The capacity of MPEG-hexPLA micelles to penetrate into primary corneal cells was investigated. The uptake into corneal cells is essential for CyA accumulation and action in the cornea, from which CyA could be released over time. Because there is no satisfactory animal model available that can reproduce globally the complexity of dry eye 
disease, ${ }^{17}$ the novel formulation was investigated in vivo in several tests to determine the formulation's impact on several ocular surface properties that are relevant to dry eye. The Schirmer test is an evaluation of the basal and reflex tear secretion, and the isotonicity test is an indication of the lachrymal fluid modifications. ${ }^{18}$ Both are currently used in the diagnosis of dry eye, and here they were used to evaluate possible modifications of the ocular surface by the micelle formulation. The pharmacokinetics of tear fluid was studied to determine the available CyA concentration in the precorneal surface after a single instillation. The quantitative corneal uptake after topical administration was studied in vivo in rat eyes. For all these in vivo evaluations, a $0.05 \%$ CyA/MPEGhexPLA micelle formulation was compared with the marketed formulation Restasis at the same CyA concentration.

\section{Materials AND Methods}

\section{Materials}

MPEG (molecular weight $=2000 \mathrm{~g} / \mathrm{mol}$ ) was purchased from Union Carbide Corporation (Texas City, TX). $\alpha$-Hydroxyoctanoic acid, 3,6dihexyl-1,4-dioxane-2,5-dione (dihexyl-substituted lactide, hexLA), and MPEG-hexPLA were synthesized as described previously. ${ }^{19,20}$ The fluorescent Nile Red-labeled MPEG-hexPLA was synthesized in the laboratory (Trubtsyn G. et al. 2011, submitted).

The following products were purchased from these companies:

Sigma-Aldrich (Munich, Germany): stannous 2-ethylhexanoate, sucrose, bovine serum albumin fraction $\mathrm{V}$, hydrocortisone and glycerol; Biotium (Hayward, CA): 3,3-dioctadecyloxacarbocyanine perchlorate $\left(\mathrm{DiOC}_{18}(3)\right)$; Invitrogen (Carlsbad, CA): serum-free keratinocyte medium, pituitary extract, recombinant epidermal growth factor, $0.05 \%$ trypsin-EDTA; Vector Laboratories (Burlingame, CA): fluorescein-labeled lectin I/isolectin B4; Abcam (Cambridge, MA): mouse monoclonal antibody to cytokeratin $3+12$; Roche (Basel, Switzerland): fibronectin; Cellial Technologies (Lens, France): rat tail collagen; Novartis (Basel, Switzerland): 0.4\% oxybuprocaine eye drops; Théa (Cleremont-Ferrand, France): dina strip Schirmer plus; Allergan (Irvine, CA): Restasis; Dynapharm Distribution (Geneva Switzerland): cyclosporin A; Fluka (Munich, Germany): analytical grade acetone, ethanol; VWR (Fontenay-sous-Bois, France): methanol for HPLC; Biosolve (Valkenswaard, The Netherlands): methanol for UPLC/MS MilliQ water was used in each experiment.

Tear osmolarity test cards were a kind gift from Tear Lab Corporation (San Diego, CA)

\section{Methods}

For the precorneal kinetics evaluation, Schirmer test, and isotonicity test, New Zealand rabbits, female, weighing $4-5 \mathrm{~kg}$, were used. Before starting the experiments, a visual evaluation of any corneal surface damage as well as fluorescein tests were performed to ensure the nonobstruction of the naso-lachrymal drainage system. The experimental protocol was approved by the Canton of Geneva's Local Ethics Committee for Animal Experimentation (1020/325/1).

For the ocular distribution experiment, 12 female, 8-week-old, 150$200 \mathrm{~g}$ Lewis rats were used. The experimental protocol was in accordance with the European Committee Directives (authorization numbers: A 75-06-12 and 75-580). All animals were treated in accordance with the ARVO Statement for the Use of Animals in Ophthalmic and Vision Research. ${ }^{21}$

\section{Preparation and Characterization of the Polymeric Micelle Formulation}

Micelle formulations were prepared as described previously by a solvent evaporation method. ${ }^{16,22}$ Briefly, $40 \mathrm{mg}$ of MPEG-hexPLA copolymer and $8.8 \mathrm{mg}$ of CyA were dissolved in $2.0 \mathrm{~mL}$ acetone. This organic phase was added dropwise under probe sonication (Digital Sonifier 450, Branson, Danbury, CT) to $4.0 \mathrm{~mL}$ of $10 \mathrm{mM}$ phosphate buffer with $10 \%$ sucrose. The acetone was then evaporated under vacuum. The obtained micelle formulation had a final copolymer concentration of $10 \mathrm{mg} / \mathrm{mL}$. After CyA quantification, the formulation was diluted with the isotonic $10 \mathrm{mM}$ phosphate buffer solution to obtain a final CyA concentration of $0.05 \%$. The fluorescent micelle formulation was prepared with the same method and had a final copolymer concentration of $20 \mathrm{mg} / \mathrm{mL}$, with a 12:88 weight ratio between the Nile Red-labeled MPEG-hexPLA and MPEG-hexPLA, and $3 \% \mathrm{w} / \mathrm{w} \mathrm{DiO}$ was loaded inside. $\mathrm{DiO}$ was used here as a model drug for CyA. The formulations were characterized in terms of size, morphology, and drug loading using dynamic laser scattering (Zetasizer HS 3000, Malvern Instruments, Malvern, UK), transmission electron microscopy (FEI Tecnai G2 Sphera, Hillsboro, OR), and high-pressure liquid chromatography (Waters, Montreux-Chailly, Switzerland), respectively, as described in previous studies. ${ }^{16,22}$ All formulations were filtered under sterile conditions before use.

\section{In Vitro Cellular Test}

Two different cell cultures were carried out: (1) Rabbit primary corneal cells were isolated from the corneas of two animals by trypsinization of both sides (epithelium and endothelium). The harvested cells were seeded into a $60-\mathrm{mm}$ tissue culture dish coated with $0.01 \mathrm{mg} / \mathrm{mL}$ fibronectin, $0.03 \mathrm{mg} / \mathrm{mL}$ rat tail collagen, and $0.01 \mathrm{mg} / \mathrm{mL}$ bovine serum albumin type $\mathrm{V}$. The cells were cultured with keratinocyte serum-free medium supplemented with $1 \%$ penicillin/streptomycin, $500 \mathrm{ng} / \mathrm{mL}$ bovine pituitary extract, $5 \mathrm{ng} / \mathrm{mL}$ recombinant epidermal growth factor, and $500 \mathrm{ng} / \mathrm{mL}$ hydrocortisone, as recommended by the supplier, in a humidified atmosphere at $37^{\circ} \mathrm{C}$ with $5 \% \mathrm{CO}_{2}$.

(2) Whole corneas were kept in culture under the same conditions, and cells from the corneas were able to migrate out of the tissue to form a mixed culture of corneal cells. At maximum two passages were performed for each culture, and cells were used after approximately 2 weeks of culture.

Identification of Corneal Cells by Immunocytochemistry. To characterize these corneal cells, immunocytochemistry was performed using an anti-cytokeratin $3+12$ antibody and fluoresceinlabeled lectin I/isolectin B4, which specifically recognized epithelial and endothelial cells, respectively. For this purpose, cells were first fixed with cold absolute ethanol at $-20^{\circ} \mathrm{C}$ for 10 minutes and then permeabilized by $0.1 \%$ Triton X100-PBS solution at room temperature for 10 minutes. Cells were subsequently incubated with 3\% BSA-PBS for 30 minutes prior to incubation at $4^{\circ} \mathrm{C}$ with the monoclonal anticytokeratin $3+12$ antibody (2Q1040) in a PBS solution containing 3\% BSA overnight. Finally, the cells were incubated at room temperature for 1 hour with the appropriate combination of fluorescently labeled secondary antibody and/or with fluorescein lectin I/isolectin B4 in PBS with 3\% BSA. Each step was followed by washes with PBS. For all stainings, cells were finally mounted on glass microscopic slides using Glycergel mounting medium (Dako, Glostrup, Denmark). Slides were then observed with an AXIO Imager Z1m fluorescence microscope (Zeiss, Feldbach, Switzerland). Images were taken using a digital Axiocam MRm camera (Zeiss). A magnification of $63 \mathrm{X}$ was used for all observations.

Micelle Uptake by Primary Rabbit Corneal Cells. To evaluate cellular uptake, a fluorescent micelle formulation with a copolymer concentration of $3 \mathrm{mg} / \mathrm{mL}$ was used. Culture medium was removed, and the cells were incubated with the micelle solution diluted $1 / 6$ in culture medium and then incubated at $37^{\circ} \mathrm{C}$ in a humidified atmosphere with $5 \% \mathrm{CO}_{2}$ for 1 hour or 24 hours. After the desired incubation time, the micelle solution was removed, and cells were washed twice with culture medium and incubated with Hoechst solution for 5 minutes to stain the nuclei. Living cells were then immediately observed by an integrated system for advanced widefield fluorescence imaging and analysis (AF6000, Leica, Germany) and, for 
the cells incubated 24 hours, also by confocal microscopy with a 510 Meta microscope (Zeiss).

\section{CyA Kinetics in Lachrymal Fluid after Topical Instillation}

For kinetics experiments, six rabbits were treated with the CyA/MPEGhexPLA micelle formulation and six others with Restasis for comparison. Twenty-five microliters of the formulation was instilled in the right eye of each rabbit with a calibrated micropipette (Assipettor-digital, Assistent, Sondheim, Germany). Two microliters of tear fluid was withdrawn at each time point using a $2-\mu \mathrm{L}$ microcap capillary (Drummond Scientific Co., Broomall, PA). The time points were $0,1,3,6,8,10,12,14,16,18,20,30,60,90,120,150$, and 180 minutes after instillation. Each $2-\mu \mathrm{L}$ aliquot was diluted in a vial containing $50 \mu \mathrm{L}$ of methanol and $100 \mathrm{ppb}$ of deuterated CyA as an internal standard. The vials were vortexed and analyzed by UPLC/ MS_MS. ${ }^{23}$ The CyA half-life, maximal concentration, and area under the curve were calculated for both formulations with WinNonlin 5.2 version software.

\section{Evaluation of the Basal Tear Production after Topical Instillation}

The basal tear production was measured by a Schirmer test carried out on six rabbits for each formulation, the CyA/MPEG-hexPLA micelle formulation and Restasis. A single instillation of $25 \mu \mathrm{L}$ was done in the right eye, and the test was performed before and after 1, 2, 4, 6, 24, and 48 hours of instillation. Topical anesthesia was performed by instillation of 1 drop of $0.4 \%$ oxybuprocaine solution 5 minutes before the insertion of the Schirmer test strip in the cul-de-sac. The time of the test was 5 minutes. The height of the Schirmer test strip wetted by the tears was measured in millimeters and plotted versus time.

\section{Evaluation of Tear Osmolarity after Topical Instillation}

Tear osmolarity was measured using a TearLab Osmolarity System (TearLab Corporation, San Diego, CA) equipped with osmolarity test cards. The instrument is designed to withdraw a volume of $50 \mathrm{~nL}$ of tears. The osmolarity was measured before and after 30, 60, and 120 minutes instillation of a $25-\mu \mathrm{L}$ formulation containing $0.05 \%$ CyA/MPEGhexPLA or Restasis. Three animals were used for each formulation.

\section{CyA Ocular Distribution after Topical Instillation}

The ocular distribution of the CyA micelle formulation and Restasis was evaluated in six rats per formulation. Two instillations per day were performed for 5 days. After sacrifice with $\mathrm{CO}_{2}$, the cornea, iris-ciliary body, aqueous humor, and vitreous were recuperated. The blood was recovered directly from the heart with a syringe, collected in a $2-\mathrm{mL}$ vial, and stored at $4^{\circ} \mathrm{C}$ for 30 minutes before centrifugation at $9000 \mathrm{~g}$ for 15 minutes. The obtained serum was kept at $-80^{\circ} \mathrm{C}$ until further analyses. The cornea, iris-ciliary body, aqueous humor, and vitreous of both eyes (treated and contralateral) were stored under the same conditions in protected vials. For the analyses, the tissues were thawed at room temperature, weighed, manually grinded, and treated as follows: $100 \mu \mathrm{L}$ of serum was diluted with $400 \mu \mathrm{L}$ of methanol containing $100 \mathrm{ppb}$ of deuterated CyA as internal standard; and $200 \mu \mathrm{L}$ of methanol containing internal standard was added to the cornea, aqueous humor, iris-ciliary body, and vitreous samples. After stirring for 12 hours, the samples were centrifuged and $150 \mu \mathrm{L}$ of the supernatant was transferred in UPLC vials. The CyA was quantified by UPLC/MS_MS.

\section{Statistics}

For all comparisons, the Mann-Whitney test was used (unpaired samples). Samples were significantly different from each other when $P<0.05$.

\section{Results}

\section{Preparation and Characterization of Polymeric Micelles}

The CyA micelle formulation used for all the studies had the following characteristics: $\mathrm{pH} 7.4 \pm 0.1$, isotonicity $318 \pm 1$ $\mathrm{mmol} / \mathrm{kg}$, and CyA concentration $0.50 \pm 0.01 \mathrm{mg} / \mathrm{mL}$, with a copolymer concentration of $3 \mathrm{mg} / \mathrm{mL}$. The micelles had a hydrodynamic diameter of $35 \pm 1 \mathrm{~nm}$, with a polydispersity index of $0.200 \pm 0.013$. The fluorescent micelle formulation had the same $\mathrm{pH}$ and isotonicity as the CyA formulation and a micelle size of $32 \pm 1 \mathrm{~nm}$ with a PI of $0.453 \pm 0.044$.

\section{In Vitro Cellular Tests}

Immunocytochemistry showed that different cell types were present when the corneas were cultured. After incubation with the anti-cytokeratin $3+12$ antibody and with fluorescein lectin I/isolectin B4, epithelial cells were only stained by the anti-cytokeratin $3+12$ antibody (Fig. 1, left side), and endothelial cells were stained only by the fluorescein lectin I/isolectin B4 (Fig. 1, right side).

The epithelial and endothelial corneal cells obtained by cornea culture were used for cellular uptake experiments. The micelle formulation was prepared with a Nile red-labeled copolymer and loaded with $\mathrm{DiO}$, which fluoresces green. Intact micelles carrying the model drug were visible by the yellow fluorescence due to the colocalization of red and green fluorescence. When the micelle released the model drug DiO, green fluorescence was observed.

After 1 hour of incubation with the fluorescent micelle formulation, yellow spots were present in the cells (Figs. 1a, 1b). Thus, micelles carrying the model drug DiO were able to interact with the cellular membrane and to penetrate into the cells. After 24 hours of incubation, a yellow fluorescence was still detectable, but at a lower intensity (Figs. 1c, 1d). In addition, green fluorescence was visible, suggesting that the micelles started to release the model drug inside the cells.

The observation of cells by confocal microscopy after incubation with the fluorescent micelle formulation for 24 hours showed the presence of green, red, and yellow fluorescence at the same level as the blue fluorescence (nuclei), meaning that micelles were able to penetrate into the corneal cells (Fig. 3).

\section{CyA Kinetics in Lachrymal Fluid after Topical Instillation}

The precorneal kinetics was evaluated in rabbit eyes after one instillation of the CyA micelle formulation or Restasis. The

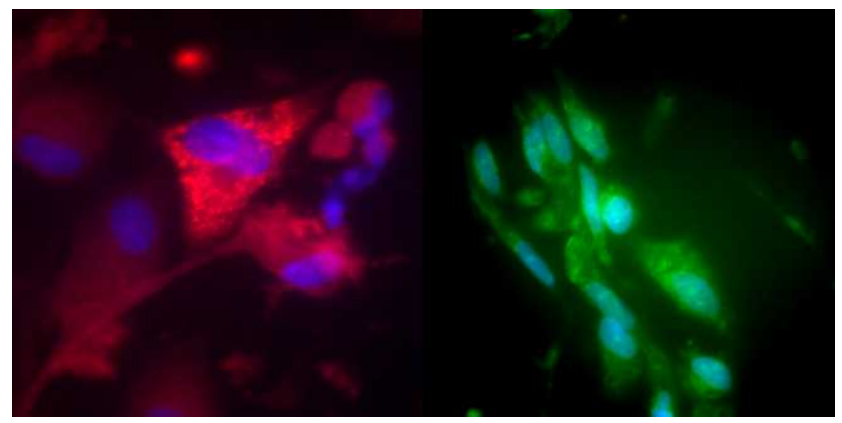

Figure 1. Corneal cells stained with monoclonal anti-cytokeratin $3+12$ antibody (red) and endothelial cells stained with fluorescein lectin I/isolectin B4 (green). 

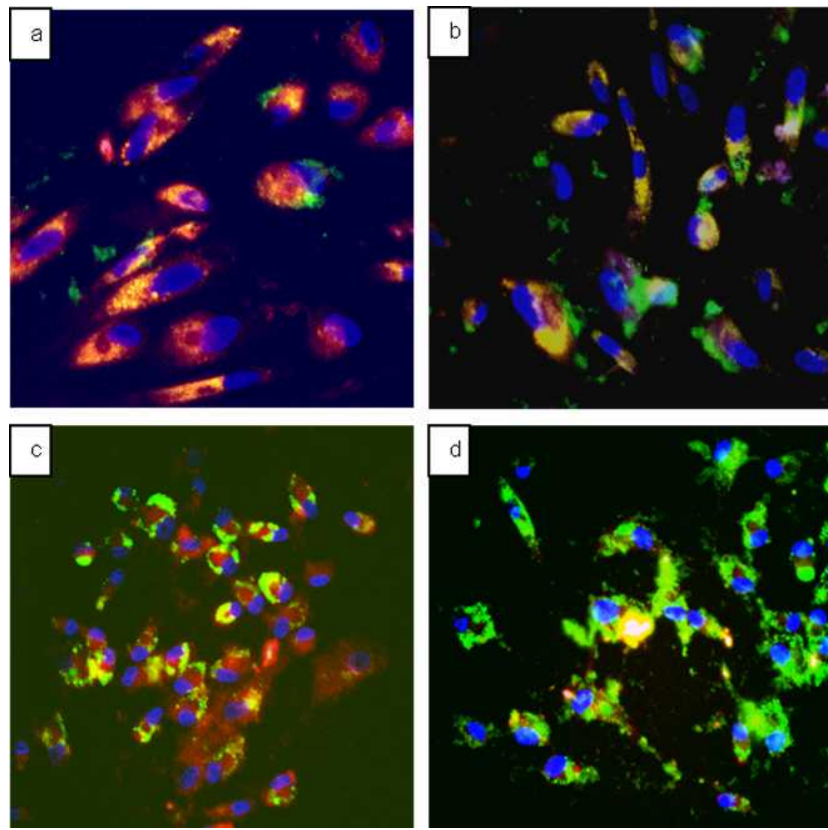

Figure 2. Corneal cells treated with the micelle formulation observed by fluorescence widefield microscopy. (a, b) After 1 hour. (c, d) 24 hours of incubation (blue fluorescence $=$ nuclei stained with Hoechst solution; yellow fluorescence $=$ DiO-loaded fluorescent micelles; red fluorescence $=$ micelle prepared with labeled copolymer; green fluorescence $=\mathrm{DiO}$ ).

obtained CyA concentrations in the lachrymal fluid are presented in Fig. 4. For both formulations, the elimination profile of CyA from the tears after topical instillation was biphasic (Fig. 4a), with a rapid elimination of the drug within the first 20 minutes (Fig. 4b) and slower thereafter (Fig. 4c). No significant difference in kinetics was found between the two formulations.

The AUC, $\mathrm{C}_{\max }$, and half-life are reported in Table 1. All three values were higher for the micelle formulation, although they were not significantly different from those of Restasis.

\section{Evaluation of Basal Tear Production after Topical Instillation}

The Schirmer test was performed in rabbit eyes to evaluate the tear secretion after topical instillation of $25 \mu \mathrm{L}$ of either formulation. The initial mean values (time zero) were between $9.8 \mathrm{~mm}$ and $10.6 \mathrm{~mm}$ for all animals. Even though no significant difference was found, it was noticed for the rabbits treated with the micelle formulation that the tear production was always higher than the baseline, except after 4 hours, when it was equal to the baseline. For the eyes treated with Restasis, more fluctuations were visible. For both formulations, the value after 48 hours was similar to the baseline value.

\section{Evaluation of Tear Osmolarity after Topical Instillation}

The initial isotonicity values of tears were $347 \mathrm{mOsm} / \mathrm{kg}$ for eyes treated with the micelle formulation and $366 \mathrm{mOsm} / \mathrm{kg}$ for eyes treated with Restasis. At all time points studied, no significant variations from the initial mean values were observed for both formulations.

\section{CyA Ocular Distribution Study after Topical Instillation}

The ocular distribution of CyA was examined in rat eyes after topical instillation of the CyA micelle formulation or Restasis twice per day for 5 days. The mean CyA concentration in the corneas of the animals treated with the CyA/MPEG-hexPLA

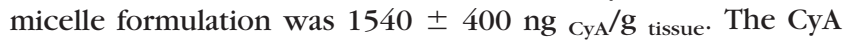
concentrations in aqueous humor and the iris-ciliary body were below $2 \mathrm{ng} / \mathrm{mL}$, which was the limit of quantification of the analytical method used. Only in two eyes of the six treated with the micelle formulation was the CyA concentration in the vitreous higher than $2 \mathrm{ng} / \mathrm{mL}$.

In the eyes treated with Restasis, the CyA concentration was higher than $2 \mathrm{ng} / \mathrm{mL}$ only in the cornea and vitreous of one out of six rats. In all other ocular tissues, the CyA concentration was below the LOQ.

In all blood samples, the CyA concentration was below the LOQ of $2 \mathrm{ng} / \mathrm{mL}$.

\section{Discussion}

The bioavailability of drugs is reduced by two principal factors: instability in the biological environment and limited transport across biological membranes. ${ }^{24} \mathrm{~A}$ carrier that can protect the drug, rapidly pass through epithelia, and execute a sustained drug release after administration is an attractive solution to improve drug bioavailability. Nano-sized colloidal systems have both abilities. In fact, the particle size is one key factor for passing through biological membranes, and nano-sized particles penetrate the corneal layers through a transcellular pathway. ${ }^{25}$ In addition, some colloidal systems have shown improved drug protection in physiological conditions. ${ }^{26,27}$ In the last decade, many colloidal systems have been developed for ophthalmic application. ${ }^{28}$ For example, liposomes and PLGA (poly(lactic-co-glycolic acid)) nanoparticles were able to prolong the retention at the corneal site for the hydrophobic drugs indoxole ${ }^{29}$ and sparfloxacin, ${ }^{30}$ respectively. Solid lipid nanoparticles, based on glyceryl dibehenate as the lipid phase and poly(ethylene)-poly(propylene) glycol and polysorbates as the aqueous phase, have been proposed as a topical ocular carrier for CyA. ${ }^{31}$ This system was efficient in solubilizing CyA and in increasing the corneal permeation in ex vivo studies on pig corneas compared to a $0.05 \%$ CyA suspension used as a reference. Among the reported colloidal systems, polymeric micelles have interesting and promising characteristics for ophthalmic applications, such as their simple preparation and nano size, the transparency and the stability of their formulations, their efficiency in drug loading, and localized drug release.

In this study, polymeric micelles were used as colloidal carriers for ocular delivery of CyA after topical instillation for dry eye treatment. A commercial emulsion, Restasis, was used as reference in all the in vivo experiments. Restasis can improve moderate and severe dry eye symptoms. ${ }^{3}$ Restasis is an anionic castor oil-in-water emulsion stabilized by polysorbate $80 .{ }^{32,33}$ Even though the exact mechanism of CyA action in the eye is not totally clear, this formulation is effective for treating keratoconjunctivitis sicca. Some local side effects, probably due to the castor oil, are reported, such as burning and stinging sensations, conjunctival hyperemia, and eye pain. ${ }^{12,34}$ The comfort for the patient can be increased by the use of a novel micelle formulation that is well tolerated. ${ }^{13,16}$

Considering that nano-sized particles are in the same size range as membrane receptors, proteins and other biomolecules, they have the ability to interact with cellular barriers, ${ }^{35}$ 


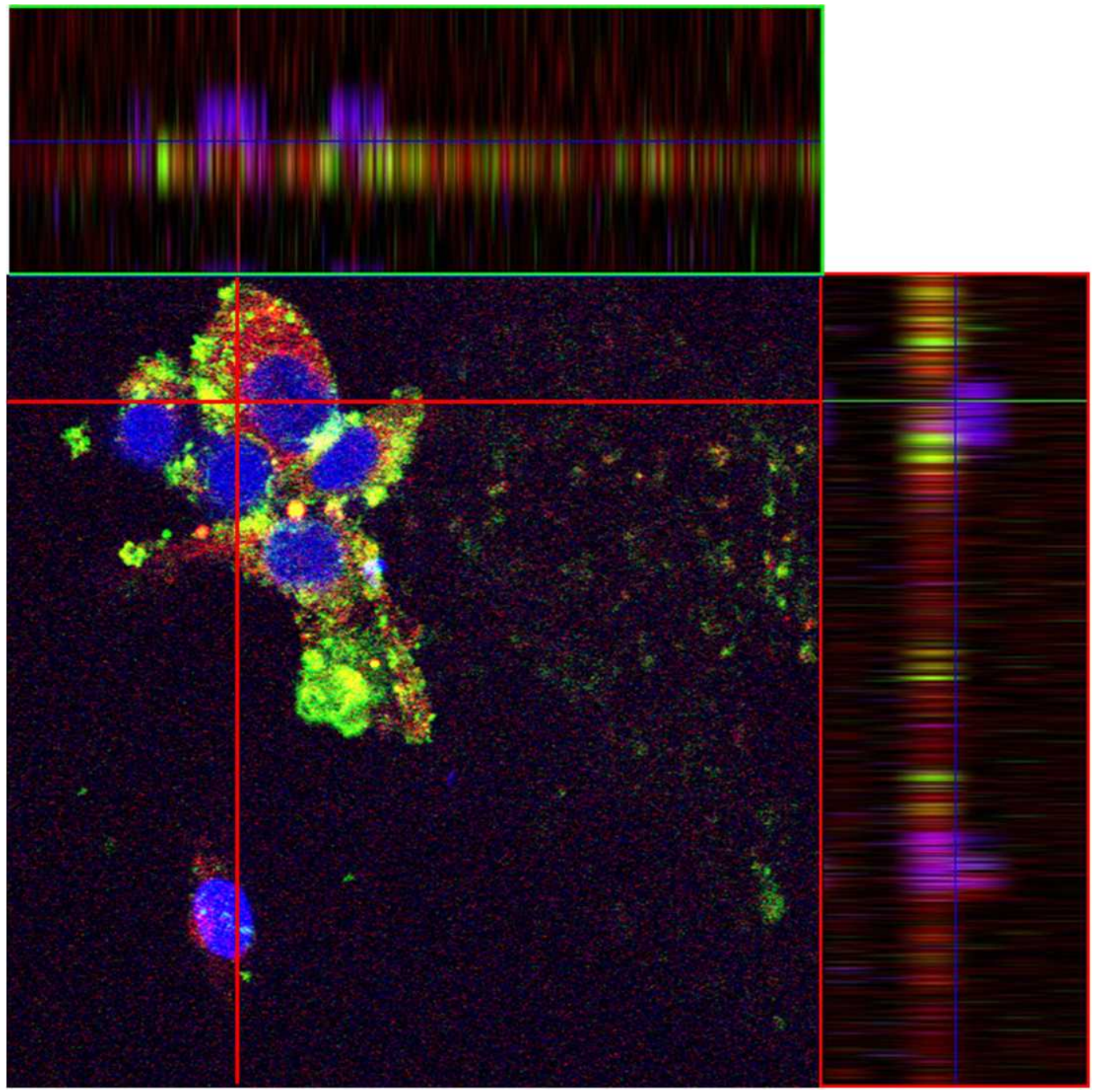

FiguRE 3. Orthogonal section of corneal cells treated with the micelle formulation observed by confocal fluorescence microscopy after 24 hours of incubation (blue fluorescence $=$ nuclei stained with Hoechst solution; yellow fluorescence $=$ DiO loaded fluorescent micelles; red fluorescence $=$ micelle prepared with labeled copolymer; green fluorescence $=\mathrm{DiO}$ ).

which is important because the cellular uptake is the first step for the drug's therapeutic action. For the treatment of ocular surface diseases, such as dry eye, the ideal case would be the uptake and the accumulation of the colloidal carriers containing CyA into corneal cells, to form a drug depot from which CyA would be released over time. To verify if the MPEGhexPLA micelles have this important carrier ability, corneal primary cells were chosen because the corneal cells come in contact with the formulation just after the instillation. Possibly, this general evaluation could also apply to conjunctival cells, which have similar characteristics and work as a barrier like the corneal cells. The results show that the micelles were internalized by the corneal cells and delivered the incorporated model drug to the interior of the cells. Primary cells were chosen also because they can provide further evidence of the nontoxicity of the micelle formulation in vivo. In fact, primary cells are more sensitive to toxic compounds than immortalized cell lines. After 24 hours of incubation with the micelle formulation, the cells were alive, meaning that these carriers did not alter the cell structure or cause irreversible damage. This provides additional evidence of the biocompatibility that has already been demonstrated for this colloidal formulation. ${ }^{13}$
The CyA micelle formulation was evaluated in vivo in terms of nonalteration of ocular surface equilibrium and its ability to deliver CyA in therapeutic concentrations to lachrymal fluid and surface ocular structures that are involved in dry eye syndrome. The composition and the volume of tears are key factors for a healthy ocular surface. Indeed, high film stability provides a first line of protection to the eye and is essential to avoid evaporation and to maintain coverage of the surface of the eye during blinking. ${ }^{36}$ In dry eye, the characteristics of the ocular surface are altered due to the reduction of tear production and/or the abnormal evaporation of tear film. Since none of the existing animal models seems to be representative of the complexity and chronicity of this multifactorial disease ${ }^{17}$ or to be suitable for assessing the efficacy of a topical formulation, two classical tests for the evaluation of the tear film were performed to investigate if the micelle formulation would negatively interact with tears or the ocular surface. A negative impact on any ocular surface properties would cause modifications to the ocular surface, such as reduced quantity and quality of the lipid tear layer, altered tear composition, and ocular surface inflammation. ${ }^{37}$ Many tests of tear film stability are available to diagnose dry eye syndrome. Among them, the Schirmer test is the simplest test 


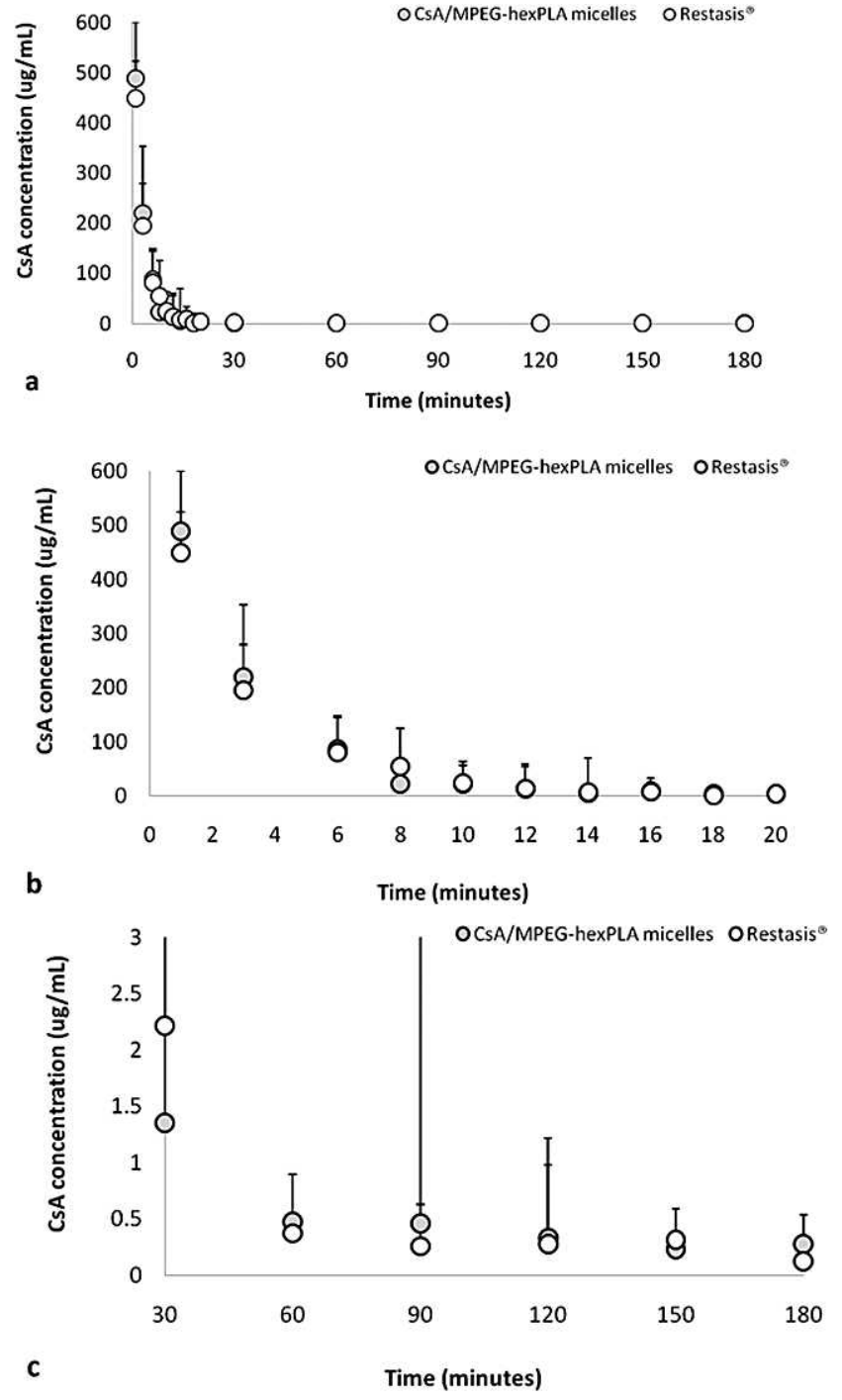

Figure 4. CyA concentrations in lachrymal fluid of rabbit eyes after a single instillation of either formulation. Values \pm SD. (a) CyA kinetics over 180 minutes. (b) CyA kinetics over 20 minutes (first elimination phase). (c) CyA kinetics between 30 and 180 minutes (second elimination phase).

to assess the basal and reflexive tear production, and the isotonicity measurement is a reproducible test that is the best indicator of dry eye. ${ }^{1,2}$ Thus, in the present study, the Schirmer test and the osmolarity measurement were chosen for the evaluation of tear film and ocular surface problems after instillation of the micelle formulation or Restasis.

The Schirmer test was performed with topical anaesthesia to evaluate the basal tear secretion. The results show that the micelle formulation did not alter the basal tear production. To evaluate if the micelle formulation increased tear evaporation, osmolarity measurements were also performed, as osmolarity is an important parameter for tear electrolyte and sugar balance.

TABle 1. CyA Pharmacokinetic Values in Lachrymal Fluid of Rabbits after a Single Instillation of Micelle Formulation or Restasis $(n=6)$.

\begin{tabular}{lccc}
\hline & AUC $\left(\mathbf{m i n}^{*} \boldsymbol{\mu g} / \mathbf{m L}\right)$ & $\mathbf{C}_{\mathbf{m a x}}(\boldsymbol{\mu g} / \mathbf{m L})$ & Half-life (min) \\
\hline Micelles & $2339 \pm 1032$ & $478 \pm 111$ & $36 \pm 9$ \\
Restasis & $2321 \pm 881$ & $451 \pm 74$ & $28 \pm 9$ \\
\hline
\end{tabular}

No alteration of tear osmolarity was observed after instillation. Restasis yielded the same results in both tests. These results imply that in healthy rabbit eyes, as used in this study, the micelle formulation did not negatively affect the tear production or the electrolyte concentration and had no influence on the basal ocular conditions, similar to the reference product.

After topical instillation in the eye, two processes take place: elimination from the ocular surface and absorption by ocular tissues. The ability of the micelles to deliver CyA to lachrymal fluid and the cornea, which are the ocular surface structures involved in ophthalmic surface diseases, was evaluated in rabbit and in rat eyes, respectively.

Concerning the kinetics of CyA in the lachrymal fluid, for both tested formulations, there was a biphasic elimination profile (Fig. 1a). The first elimination phase (Fig. 4b) was rapid, lasting approximately 20 minutes; and the second elimination phase was slower (Fig. 4c). The rapid elimination (typically 1-2 minutes) $)^{27,38}$ of an excess volume of instilled liquid from the surface of the eye is due to naso-lachrymal drainage, tear turnover, and protein binding. ${ }^{39}$ When the volume in the cul-desac reverts to the basal value, the elimination of the drug from the eye surface slows down with the normal tear turn-over (Fig. 4c), which corresponds to $7 \%$ per minute in rabbit eyes. ${ }^{40}$ The precorneal kinetics indicated that after the first rapid elimination phase, 3 hours after the instillation, a CyA concentration of $327 \pm 262 \mathrm{ng} / \mathrm{mL}$ for the micelle formulation and of $142 \pm 70$ $\mathrm{ng} / \mathrm{mL}$ for Restasis were still present in the lachrymal fluid and were consequently available for therapeutic action.

Since the micelle formulation yielded the same results as Restasis in terms of alterations of ocular surface properties and of the CyA concentration delivered to lachrymal fluid, this novel colloidal formulation could be efficiently used to treat dry eye.

The absorption of the drug is regulated by the conjunctival and corneal barriers, both limiting the drug diffusion. The corneal absorption allows the drug to reach the corneal layers and then the anterior chamber of the eye. The conjunctival absorption diverts the majority of the drug to the systemic circulation because it is a highly vascularized membrane. A certain amount of the drug can be absorbed by the inner tissues of the conjunctiva and reach the posterior segment of the eye by the trans-scleral route. ${ }^{41}$ This evaluation showed the ability of the micelle carriers to deliver CyA into corneal layers. The results obtained for the CyA concentrations in tears and in ocular tissues after topical instillation can be qualitatively correlated even though these experiments were performed in two different animal species because both are routinely used as models for ocular investigations and both animals are used in some dry eye models. ${ }^{17}$ The ocular distribution experiment in rats showed another interesting feature of the micelle carriers. The CyA concentration found in the corneas of animals treated with the colloidal system was above the therapeutic CyA range of concentrations (50-300 $\mathrm{ng}_{\mathrm{CyA}} / \mathrm{g}_{\text {tissue }}$ ), with a value of 1540

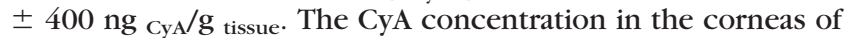
the rats treated with Restasis was below $2 \mathrm{ng} / \mathrm{mL}$, corresponding to the LOQ of the analytical method. The micelles can deliver a high drug amount to the cornea, which becomes a drug depot, releasing the drug over time. This effect is not visible in a short period of time, since the CyA concentration in tears was not significantly different after 3 hours for both formulations. However, with the micelle formulation, the number of instillations per day can be reduced by increasing the patient's compliance. Furthermore, recent studies by De Paiva et al. ${ }^{42}$ suggested that with the dry eye disease, a primary autoimmune reaction occurs not only in the conjunctiva but also in the cornea. The cornea can be a suitable target for dry eye treatment, whereby a suppression of the immune reaction will significantly improve the protective function of the corneal epithelial barrier. 


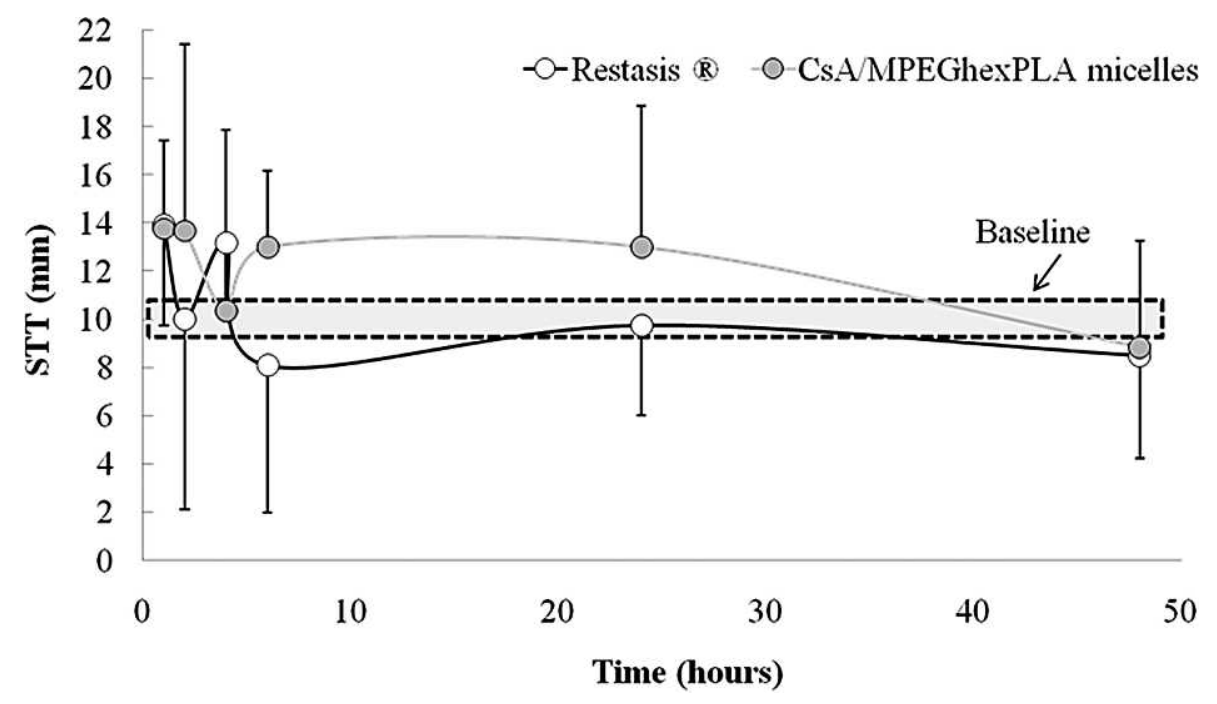

FiguRE 5. Mean values of tear secretion obtained after a single instillation of the micelle formulation or Restasis $(n=6)$. The area with the dashed line indicates the initial tear production before instillations.

Finally, it should be noted that after topical instillation, a large amount of the applied drug can be systemically absorbed, after which systemic side effects can occur. The CyA concentrations in the bloodstream were quantified after instillation of both formulations, and they were below $2 \mathrm{ng} /$ $\mathrm{mL}$. No systemic absorption of the drug at a therapeutic level was observed. Hence, the topical treatment with this micelle formulation or Restasis can avoid systemic CyA side effects.

Both formulations showed no alteration of ocular surface properties (Fig. 5) and precorneal kinetics parameters of CyA. However, the nature of the formulation is an important factor influencing CyA tolerance, availability, and elimination profile. ${ }^{43}$ This study compared an aqueous clear micelle solution to an oil-in-water opaque emulsion. In general, lipophilic vehicles are poorly tolerated on the ocular surface, whereas hydrophilic vehicles are better tolerated reducing the discomfort reported by patients. Furthermore, the hydrophobicity of the excipients present in Restasis enhances the affinity of CyA for this hydrophobic vehicle, reducing its affinity for the hydrophilic tear layer, and then increasing its elimination from the ocular surface. This is a probable explanation for the fact that CyA was found only in the cornea of the rats treated with micelle formulation and not in the rats instilled with Restasis. In two previous studies, ${ }^{16,23}$ the ocular tolerance of Restasis and micelle formulation was assessed by confocal laser scanning ophthalmoscopy. This technique allowed the quantification of the damage for the ocular surface caused by the instilled formulation. ${ }^{44}$ Both formulations were considered to be well tolerated with a percentage of the injured ocular surface of less than $20 \% .{ }^{45}$ However, the damaged ocular surface was $18.00 \pm 6.82 \%$ in the case of Restasis compared to $5.63 \pm 2.82 \%$ for the micelle formulation. It is expected for a formulation that is less well tolerated, that the activation of protective mechanisms of the eye can increase the drug elimination and decrease the drug absorption. Thus, the presented novel formulation shows important advantages in terms of ocular tolerance and corneal penetration compared to the commercial product.

\section{Summary ANd Conclusions}

The aim of this study was the in vitro and in vivo evaluation of a novel CyA topical formulation for dry eye treatment.
This study showed the ability of MPEG-hexPLA micelles to interact with corneal primary cells and release the incorporated drug model inside the target cells. No influence on the primary cell viability was observed. The CyA micelle formulation did not affect the surface ocular properties when topically instilled in rabbit eyes, which mirrored the results of the commercial CyA emulsion used as a reference. These in vitro and in vivo surface evaluations confirm the biocompatibility of the novel micelle formulation.

The CyA concentration found in lachrymal fluid was comparable between the two formulations and showed that after a first elimination phase, CyA was still present in tears 3 hours after instillation. The CyA micelle formulation was able to efficiently deliver CyA into the cornea, reaching therapeutic concentrations after a protocol of two instillations per day for 5 days. The MPEGhexPLA micelle formulation was able to provide a selective delivery of CyA into the cornea, avoiding systemic absorption and without compromising the ocular surface stability. Furthermore, due to its patient-friendly characteristics, such as transparency, ocular tolerance and biocompatibility, the micelle formulation could be efficiently applied topically without reducing the patient's compliance. This new colloidal formulation could be efficiently applied to humans for the treatment of immunemediated ocular surface diseases for which administration of CyA is required. In particular, this novel CyA formulation is suitable for dry eye treatment.

\section{Acknowledgments}

The authors thank the Swiss National Science Foundation for financial support (SNF 200020-103752). The authors are grateful to Christoph Bauer (University of Geneva) for his help in the setup of widefield fluorescence microscopy, to Catherine Siegfried (University of Geneva) for her help during the in vivo experiments, and to Youssef Daaly (University of Geneva) for his advice on the pharmacokinetics calculations.

\section{References}

1. Gaffney EA, Tiffany JM, Yokoi N, Bron AJ. A mass and solute balance model for tear volume and osmolarity in the normal and the dry eye. Prog Retin Eye Res. 2010;29:59-78.

2. Lemp MA. Report of the National Eye Institute/industry workshop on clinical trials in dry eye. CLAOJ. 1995;21:221-232. 
3. Rao SN. Topical Cyclosporine $0.05 \%$ for the prevention of dry eye disease progression. J Ocul Pharm Ther. 2010;26:157164 .

4. Survase SA, Kagliwal LD, Annapure US, Singhal RS. Cyclosporin A. - A review on fermentative production, downstream processing and pharmacological applications. Biotechnol Adv. 2011;29:418-435.

5. Italia JI, Bhardwaj V. Kumar MN. Disease, destination, dose and delivery aspects of ciclosporin: the state of the art. Drug Discov Today. 2006;11:846-854.

6. Rubin M, Rao SN. Efficacy of topical cyclosporin $0.05 \%$ in the treatment of posterior blepharitis. J Ocul Pharmacol Ther. 2006;22(1):47-53.

7. Hingorani M, Calder VL, Buckley RJ, Lightman S. The immunomodulatory effect of topical cyclosporin A in atopic keratoconjunctivitis. Invest Ophthalmol Vis Sci. 1999;40:392399.

8. Yoon KC, Heo H, Kang IS, et al. Effect of topical cyclosporin A on herpetic stromal keratitis in a mouse model. Cornea. 2008; 27:454-460.

9. Kaswan RL. Intraocular penetration of topically applied cyclosporine. Transplant Proc. 1988;2:650-655.

10. Mondon K, Zeisser-Labouèbe M, Gurny R, Möller M. Novel Cyclosporin A formulations using MPEG-hexyl-substituted polylactide micelles: a suitability study. Eur J Pharm Biopharm. 2011;77:56-65.

11. Kunert KS, Tisdale AS, Stern ME, Smith JA, Gipson IK. Analysis of cyclosporine treatment of patients with dry eye syndrome: effect on conjunctival lymphocytes. Arch Ophthalmol. 2000; 118:1489-1496.

12. Sheppard JD, Scoper SV, Samudre S. Topical loteprednol pretreatment reduces cyclosporine stinging in chronic dry eye disease. J Ocul Pharmacol Ther. 2011;27:23-27.

13. Di Tommaso C, Behar-Cohen F, Gurny R, Möller M. Colloidal systems for the delivery of Cyclosporin A to the anterior segment of the eye. Ann Pharm Fr. 2011;69:116-123.

14. Gokce EH, Sandri G, Bonferoni MC, et al. Cyclosporine A loaded SLNs: evaluation of cellular uptake and corneal cytotoxicity. Int J Pharm. 2008;364:76-86.

15. Yenice I, Mocan MC, Palaska E, et al. Hyaluronic acid coated poly-epsilon-caprolactone nanospheres deliver high concentrations of cyclosporine A into the cornea. Exp Eye Res. 2008; 87:162-167.

16. Di Tommaso C, Torriglia A, Furrer P, Gurny R, Möller M. Ocular biocompatibility of novel Cyclosporin A formulations based on methoxy poly(ethylene glycol)-hexylsubstituted poly(lactide) micelle carriers. Int J Pharm. 2011;416:515-524.

17. Barabino S Dana MR. Animal models of dry eye: a critical assessment of opportunities and limitations. Invest Ophthalmol Vis Sci. 2004;45:1641-1646.

18. Lemp MA, Baudouin C, Baum J, et al. The definition and the classification of dry eye disease: report of the definition and classification subcommittee of the International Dry Eye Workshop (2007). Ocul Surf. 2007;5:75-92.

19. Trimaille T, Mondon K, Gurny R, Möller M. Novel polymeric micelles for hydrophobic drug delivery based on biodegradable poly(hexyl-substituted lactides). Int J Pharm. 2006;319: 147-154.

20. Trimaille T, Möller M, Gurny R. Synthesis and ring-opening polymerization of new monoalkyl-substituted lactides. J Polym Sci Part A:Polym Chem. 2004;42:4379-4391.

21. ARVO. Statement for the use of animals in ophthalmic and vision research. Invest Ophthalmol Vis Sci. 1994;35:2-5.

22. Di Tommaso C, Como C, Gurny R, Möller M. Investigations on the lyophilisation of MPEG-hexPLA micelle based pharmaceutical formulations. Eur J Pharm Sci. 2010;40:38-47.
23. Rodriguez-Aller M, Kaufmann B, Guillarme $\mathrm{D}$, et al. In vivo characterisation of a novel water-soluble cyclosporine A prodrug for the treatment of dry eye disease. Eur J Pharm Biopharm. 2011;80:544-552.

24. Alonso MJ. Nanomedicines for overcoming biological barriers. Biomed Pharmacother. 2004;58:168-172.

25. Calvo P, Thomas C, Alonso MJ, Vila Jato JL, Robinson J. Study of the mechanisms of interaction of poly-E-caprolactone nanocapsules with the cornea by confocal laser scanning microscopy. Int J Pharm. 1994;103:283-291.

26. Suri SS, Fenniri H, Singh B. Nanotechnology-based drug delivery systems. J Occup Med Toxicol. 2007;2:16.

27. Araújo J, Gonzalez E, Egea MA, Garcia ML, Souto EB. Nanomedicine for ocular NSAIDs: safety on drug delivery. Nanomedicine. 2009;5:394-401.

28. Sahoo SK, Dilnawaz F, Krishnakumar S. Nanotechnology in ocular drug delivery. Drug Discov Today. 2008;13:144-151.

29. Schaeffer HE, Krohn DL. Liposomes in topical drug delivery. Invest Ophthalmol Vis Sci. 1982;22:220-227.

30. Gupta H, Aqil M, Khar RK, Ali A, Bhatnagar A, Mittal G. Sparfloxacin-loaded PLGA nanoparticles for sustained ocular drug delivery. Nanomedicine. 2010;6:324-333.

31. Gokce EH, Sandri G, Bonferoni MC, et al. Cyclosporine A loaded SLNs: evaluation of cellular uptake and corneal cytotoxicity. Int J Pharm. 2008;364:76-86.

32. Ding S, Olejnik O, Reis B, inventors; Allergan, Inc., assignee. Emulsion eye drop for alleviation of dry eye related symptoms in dry eye patients and/or contact lens wearers. US patent no. 5,981,607. November 9, 1999.

33. Sall K, Stevenson OD, Mundorf TK, Reis BL. Two multicenter, randomized studies of the efficacy and safety of cyclosporine ophthalmic emulsion in moderate to severe dry eye disease. CyA Phase 3 Study Group. Ophthalmology. 2000;107:631639.

34. McCabe E, Narayanan S. Advances in anti-inflammatory therapy for dry eye syndrome. Optometry. 2009;80:555-566.

35. Wan ACA, Ying JY. Nanomaterials for in situ cell delivery and tissue regeneration. Adv Drug Del Rev. 2010;62:731-740.

36. Gaudana R, Ananthula HK, Parenky A, Mitra AK. Ocular drug delivery. AAPS J. 2010;12:348-360.

37. Johnson ME, Murphy PJ. Changes in the tear film and ocular surface from dry eye syndrome. Prog Retin Eye Res. 2004;23: 449-474.

38. Kaur IP, Smitha R. Penetration enhancers and ocular bioadhesives: two new avenues for ophthalmic drug delivery. Drug Dev Ind Pharm. 2002;28:353-369.

39. Gaudana R, Jwala J, Boddu SH, Mitra AK. Recent perspectives in ocular drug delivery. Pharm Res. 2009;26:1197-1216.

40. Chrai SS, Patton TF, Mehta A, Robinson JR. Lacrimal and instilled fluid dynamics in rabbit eye. J Pharm Sci. 1973;62: 1112-1121.

41. Järvinen $\mathrm{K}$, Järvinen $\mathrm{T}$, Urtti A. Ocular absorption following topical delivery. Adv Drug Deliv Rev. 1995;16:3-19.

42. De Paiva CS, Volpe EA, Gandhi NB, et al. Disruption of TGF-b signaling improves ocular surface epithelial disease in experimental autoimmune keratoconjunctivitis sicca. PloS One. 2011;6:e29017.

43. Kuwano M, Ibuki H, Morikawa N, Ota A, Kawashima Y. Cyclosporine A formulation affects its ocular distribution in rabbits. Pharm Res. 2002;19:108-111.

44. Furrer P, Plazonnet B, Mayer JM, Gurny R. Application of in vivo confocal microscopy to the objective evaluation of ocular irritation induced by surfactants. Int J Pharm. 2000;207:8998.

45. Kaelin P. "Contribution à la validation d'un test de tolérance oculaire sur la souris."Université de Lausanne, 1994;119. 